\title{
Cooperative Effects in Models of Steady-State Transport across Membranes, I*
}

\author{
Terrell L. Hill and Yi-der Chen
}

DIVISION OF NATURAL SCIENCES, UNIVERSITY OF CALIFORNIA (SANTA CRUZ)

\author{
Communicated January 19, 1970
}

\begin{abstract}
Three simple models are discussed which show cooperative effects in the steady-state transport of neutral molecules across a membrane. The Bragg-Williams approximation is employed, and its limitations discussed. In this approximation to cooperative problems, the diagram method for the calculation of steady-state probabilities and fluxes can still be used. This paper is closely related to one by Blumenthal, Changeux, and Lefever, and provides the foundation for sequels on an oscillatory steady-state phase-transition model (part II) and on simple models possibly related to steady-state potassium ion transport across a nerve membrane (part III).
\end{abstract}

Introduction. In this paper and its sequels (parts II and III), we give a preliminary report on work in progress in the general area of cooperative effects in steady-state transport across membranes. Although we have been interested in this subject for some time,$^{1-4}$ the immediate stimulus for the present work came from the very interesting paper by Blumenthal, Changeux, and Lefever. ${ }^{5}$ The excellent and closely related work by Adam $^{6}$ should also be mentioned.

As in reference 5, the present paper (part I) is confined to models for the transport of neutral molecules. In part III, consider the transport of ions, and membrane potential (electric field) effects. Our very limited objective in part III is to find simple models which can generate curves with the well-known ${ }^{7,8}$ form of those found experimentally in steady-state potassium ion transport across a nerve (squid) membrane, in the special case of high external potassium ion concentration. Whether these models are in fact related to actual ionic transport mechanisms remains to be seen.

Part I provides the necessary background for parts II and III, and establishes the connection with Blumenthal, Changeux, and Lefever. ${ }^{5}$ In part II, we discuss a rather different type of model which shows oscillatory behavior at steady state.

Blumenthal-Changeux-Lefever Model. The diagram method ${ }^{9}$ and our previous work ${ }^{10}$ provide an easy derivation of the main equations of Blumenthal, Changeux, and Lefever. This model is concerned with the transport of neutral molecules ( 0 ) between two baths ( $A$ and $B$ ), across a symmetrical membrane which consists of $M$ identical units. Each unit (protein molecule plus lipid) can be either in conformation I or II. There is no interaction between neutral molecules and a unit in conformation I, but a unit in conformation II can bind and 
transport neutral molecules (0) via the four states shown in Figure 1a: each unit of type II has two equivalent binding sites for 0 , one accessible to each bath. The transition probabilities (rate constants) between all states are introduced in Figure $1 b$. The rate constants for binding, $\alpha_{A}$ and $\alpha_{B}$, are proportional to the respective bath concentrations of 0 . The equilibrium case is $\alpha_{A}=\alpha_{B}$.

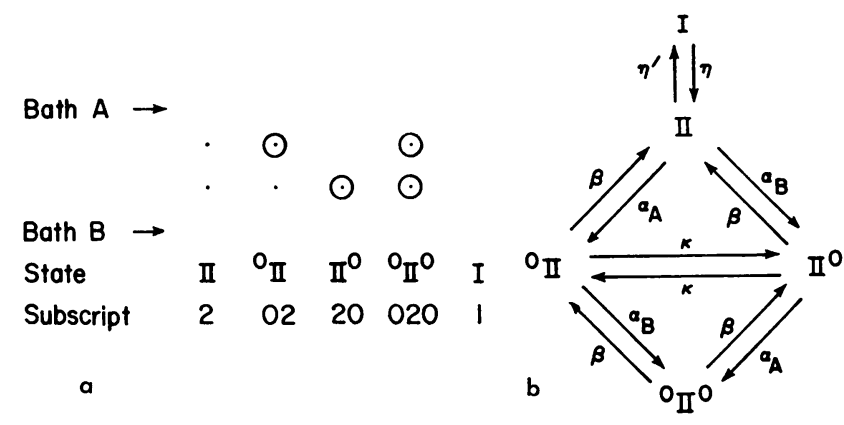

FIG. 1.-States and transition probabilities for the BlumenthalChangeux-Lefever model.

The cooperative effects we are interested in arise from interactions between neighboring units and depend on the conformation of these units. However, we omit these interactions temporarily. In the absence of interactions, the $M$ units act independently of one another.

This model, without conformation I, was investigated for another purpose in reference 10. The steady-state probabilities of the different states follow directly from equations (3) of reference 10 by appropriate ${ }^{9}$ introduction of $\eta$ and $\eta^{\prime}$ :

$$
\begin{aligned}
p_{1} & =\eta^{\prime} \beta^{2}\left(4 \kappa+2 \beta+\alpha_{\mathrm{A}}+\alpha_{\mathrm{B}}\right) / \Sigma, \\
p_{2} & =\eta \beta^{2}\left(4 \kappa+2 \beta+\alpha_{\mathrm{A}}+\alpha_{\mathrm{B}}\right) / \Sigma, \\
p_{02} & =\eta \beta\left[2 \kappa\left(\alpha_{\mathrm{A}}+\alpha_{\mathrm{B}}\right)+\alpha_{\mathrm{A}}\left(\alpha_{\mathrm{A}}+\alpha_{\mathrm{B}}+2 \beta\right)\right] / \Sigma, \\
p_{20} & =\eta \beta\left[2 \kappa\left(\alpha_{\mathrm{A}}+\alpha_{\mathrm{B}}\right)+\alpha_{\mathrm{B}}\left(\alpha_{\mathrm{A}}+\alpha_{\mathrm{B}}+2 \beta\right)\right] / \Sigma,
\end{aligned}
$$

and

$$
p_{020}=\eta\left[\left(\alpha_{\mathrm{A}}+\alpha_{\mathrm{B}}\right)^{2} \kappa+\alpha_{\mathrm{A}} \alpha_{\mathrm{B}}\left(\alpha_{\mathrm{A}}+\alpha_{\mathrm{B}}+2 \beta\right)\right] / \Sigma,
$$

where

$$
\begin{aligned}
\Sigma=\eta\left(\alpha_{\mathrm{A}}+\alpha_{\mathrm{B}}+2 \beta\right)\left[\left(\alpha_{\mathrm{A}}+\beta\right)\left(\alpha_{\mathrm{B}}+\beta\right)+\kappa\left(\alpha_{\mathrm{A}}+\alpha_{\mathrm{B}}+2 \beta\right)\right]+ \\
\eta^{\prime} \beta^{2}\left(4 \kappa+2 \beta+\alpha_{\mathrm{A}}+\alpha_{\mathrm{B}}\right) .
\end{aligned}
$$

Let $P_{2}$ be the total probability of conformation II. Then

$$
\begin{aligned}
P_{2} /\left(1-P_{2}\right) & =\left(p_{2}+p_{02}+p_{20}+p_{020}\right) / p_{1} \\
& =\frac{\left[\left(\alpha_{\mathrm{A}} / \beta\right)+1\right]\left[\left(\alpha_{\mathrm{B}} / \beta\right)+1\right]+(\kappa / \beta)\left[\left(\alpha_{\mathrm{A}} / \beta\right)+\left(\alpha_{\mathrm{B}} / \beta\right)+2\right]}{\left(\eta^{\prime} / \eta\right)\left\langle 1+\left\{4(\kappa / \beta) /\left[\left(\alpha_{\mathrm{A}} / \beta\right)+\left(\alpha_{\mathrm{B}} / \beta\right)+2\right]\right\}\right\rangle} .
\end{aligned}
$$


The net steady-state flux of 0 molecules from bath $A$ to bath $B$ is

$$
J=M_{\kappa}\left(p_{02}-p_{20}\right)
$$

or

$$
\frac{J}{M_{\kappa}}=\frac{P_{2}\left[\left(\alpha_{\mathrm{A}} / \beta\right)-\left(\alpha_{\mathrm{B}} / \beta\right)\right]}{\left[\left(\alpha_{\mathrm{A}} / \beta\right)+1\right]\left[\left(\alpha_{\mathrm{B}} / \beta\right)+1\right]+(\kappa / \beta)\left[\left(\alpha_{\mathrm{A}} / \beta\right)+\left(\alpha_{\mathrm{B}} / \beta\right)+2\right]} .
$$

Note that $\eta^{\prime} / \eta$ enters the expression for $J$ only through $P_{2}$.

We now wish to introduce interactions between units. Suppose, for example, that units of type I fit well together in a two-dimensional lattice, as do those of type II, but that a I does not fit well in a II lattice, and vice versa. The problem is then very much more complicated, even at equilibrium, ${ }^{11}$ because the units are no longer independent of each other. In fact, the entire system has to be considered a single "unit" in an exact treatment. However, it is the essence of the so-called Bragg-Williams approximation, ${ }^{11}$ to count interactions as if the units were independent. Therefore, if we are willing to use this rather rough approximation, we can include interactions between units and still use the diagram method (which assumes independent units) to obtain steady-state properties. One simply has to include interaction factors in appropriate transition probabilities in such a way as to be consistent with the Bragg-Williams approximation at equilibrium. Hence, in this approximation, equations (3) and (4) would still apply without formal change. We shall discuss further, below, the implications of the Bragg-Williams method, but proceed now to use it.

At equilibrium, if there were no interactions between units, the grand partition function per unit would be

$$
\begin{aligned}
\xi & =Q_{1}+Q_{2}+Q_{02} \lambda+Q_{20} \lambda+Q_{020} \lambda^{2} \\
& =Q_{1}+Q_{2}(1+q \lambda)^{2},
\end{aligned}
$$

where $\lambda=e^{\mu / k T}, \mu=$ chemical potential of 0 , and

$$
Q_{02}=Q_{20}, \quad q \equiv Q_{02} / Q_{2}, \quad Q_{020}=Q_{2} q^{2} .
$$

The absolute activity $\lambda$ is proportional to concentration, in a dilute solution. Then, since $p_{1}{ }^{e} / p_{2}{ }^{6}=Q_{1} / Q_{2}$ and $p_{1}{ }^{6} \eta=p_{2}{ }^{\circ} \eta^{\prime}$ (detailed balance), where $e=$ equilibrium, we must have $Q_{1} / Q_{2}=\eta^{\prime} / \eta$ for self-consistency. Similarly, we find $q \lambda=\alpha / \beta$ (at steady state, $\alpha_{A} / \beta=q \lambda_{A}$ and $\alpha_{B} / \beta=q \lambda_{B}$ ).

We now include nearest neighbor interactions, in the Bragg-Williams approximation. Let $w_{12}$ be the interaction energy between a nearest neighbor I-II pair, etc. We assume for simplicity that binding of the relatively small 0 molecule does not influence these interactions. Let $c$ be the nearest neighbor number in the lattice (e.g., $c=6$ in a close-packed lattice). Then, for given $P_{2}=1-P_{1}$, any particular unit has, on the average, $c P_{1}$ and $c P_{2}$ neighboring units of the two types. Therefore, equation (5) becomes ${ }^{12}$

$$
\xi=Q_{1} a_{11}{ }^{-P_{1}} a_{12}{ }^{-P_{2}}+Q_{2} a_{22}{ }^{-P_{2}} a_{12}{ }^{-P_{1}}(1+q \lambda)^{2},
$$

where $a_{11}=e^{c v_{11} / k T}$, etc. We still have $\alpha / \beta=q \lambda$, but now 


$$
\frac{\eta^{\prime}}{\eta}=\frac{Q_{1} a_{11}{ }^{-P_{1}} a_{12}{ }^{-P_{2}}}{Q_{2} a_{22}{ }^{-P_{2}} a_{12}{ }^{-P_{1}}}=Q a^{1-2 P_{2}}
$$

where

$$
\begin{gathered}
Q \equiv \frac{Q_{1} a_{11}-1 / 2}{Q_{2} a_{22}{ }^{-1 / 2}}, \quad a \equiv \frac{a_{12}}{a_{22}{ }^{1 / 2} a_{11}{ }^{1 / 2}}=e^{-c w / 2 k T}, \\
w \equiv w_{11}+w_{22}-2 w_{12} .
\end{gathered}
$$

The value of $Q$ (an "equilibrium constant") indicates the relative thermodynamic stability, per unit, of an all-I lattice and an all-II lattice. Cooperativity occurs when $w<0$ or $a>1$; as mentioned above, an example would be when a unit of type I is a "misfit" in a II lattice, and vice versa.

At equilibrium, from either equation (3) or (7), we have

$$
P_{2} a^{1-2 P_{2}} /\left(1-P_{2}\right)=(1+q \lambda)^{2} / Q \text {. }
$$

At steady state, using equations (3) and (8),

$$
\frac{P_{2} a^{1-P_{2}}}{1-P_{2}}=\frac{\left[\left(\alpha_{\mathrm{A}} / \beta\right)+1\right]\left[\left(\alpha_{\mathrm{B}} / \beta\right)+1\right]+(\kappa / \beta)\left[\left(\alpha_{\mathrm{A}} / \beta\right)+\left(\alpha_{\mathrm{B}} / \beta\right)+2\right]}{Q\left\langle 1+\left\{4(\kappa / \beta) /\left[\left(\alpha_{\mathrm{A}} / \beta\right)+\left(\alpha_{\mathrm{B}} / \beta\right)+2\right]\right\}\right\rangle} .
$$

If the right-hand side of either equation (10) or (11) is denoted by $y$, then $P_{2}-1 / 2$ is an odd function of $\ln y$, with an inflection point at $P_{2}=1 / 2, y=1$. This function is illustrated elsewhere. ${ }^{13}$ The critical value of $w$ is $c w / k T=-4$, or $a=e^{2}$.

We note that $\eta$ and $\eta^{\prime}$ occur in equation (3) only as the (thermodynamic) ratio $\eta^{\prime} / \eta$. Hence, it is not necessary for present purposes to decide (on kinetic grounds) how to "split" $Q a^{1-2 P_{3}}$ between $\eta$ and $\eta$ '.

Equations (11) and (4) are essentially the same as equations (2.3) and (2.5), respectively, of reference 5 . The correspondences between the two notations are:

$$
P_{2} \leftrightarrow\langle r\rangle, \alpha_{\mathrm{A}} / \beta \leftrightarrow \alpha, \alpha_{\mathrm{B}} / \beta \leftrightarrow \beta, \kappa / \beta \leftrightarrow \epsilon, a^{-2} \leftrightarrow \Lambda, a Q \leftrightarrow l .
$$

Figures 2 and 3 illustrate equations (4) and (11), in the physically important special case $\beta \gg \kappa$. Blumenthal, Changeux, and Lefever give a number of additional curves. In these figures, $\alpha_{B} / \beta$ is held constant and $\alpha_{A} / \beta$ varied, starting on the left from $\alpha_{\mathbb{A}} / \beta=0$. Although the resemblance to the experimental potassium flux curves referred to above is striking and suggestive, the analogy cannot be pushed very far because: (1) the abscissa in the figures is a concentration difference and not a membrane potential (as in the experiments); (2) the molecule being transported is not an ion; and (3) there is only one "transition" in the figures, whereas the experiments show a second (reverse) transition in the region of positive flux. All three of these shortcomings are removed in part III, incidentally.

The physical interpretation of Figures 2 and 3 is straightforward: when $\alpha_{\mathrm{A}} / \beta$ is small, conformation I (which is impermeable to 0 ) is more stable than II; an increase in $\alpha_{\mathrm{A}} / \beta$, however, shifts this relative stability to favor II (because of 
an increase in relative population of ${ }^{0} \mathrm{II}, \mathrm{II}^{0}$, and ${ }^{\circ} \mathrm{II}^{0}$ ); with a sufficiently negative value of $c w / k T$, the transition from small $P_{2}$ to large $P_{2}$ is rather sudden; since conformation II can transport 0 molecules, there is a concomitant increase in $|J|$.

The dashed line in Figures 2 and 3 shows how the flux would behave if $P_{2}$

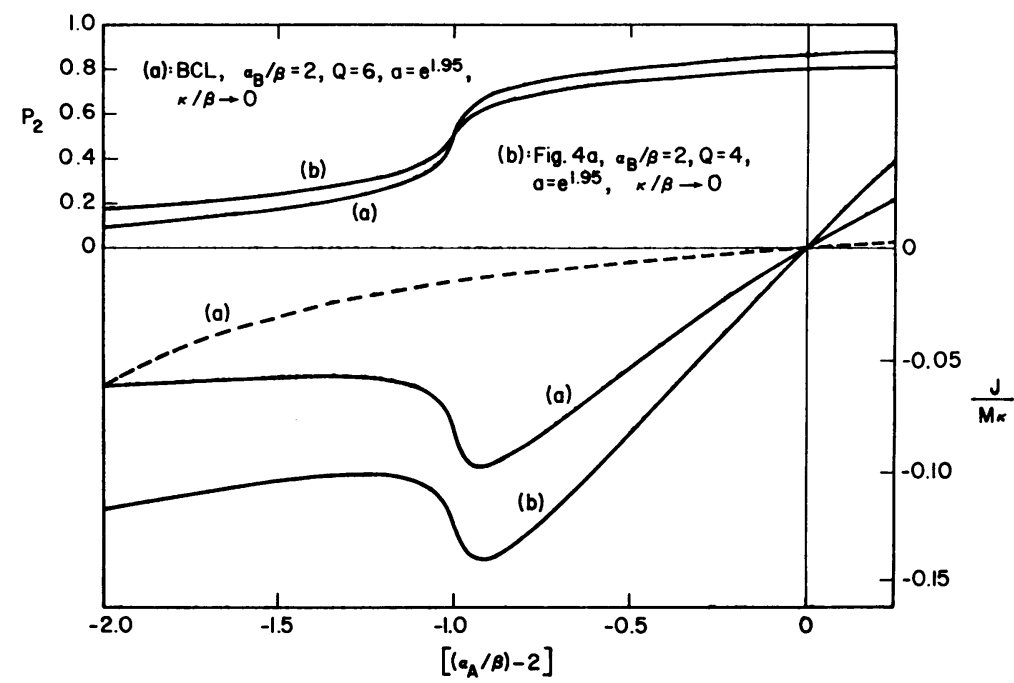

Fig. 2.-Cooperative effect on the flux when $\alpha_{B} / \beta=2$ : (a) BlumenthalChangeux-Lefever (BCL) model; (b) model in Fig. $4 a$. $Q$ chosen (arbitrarily) to give $P_{2}=1 / 2$, when $\alpha_{A}=\alpha_{B} / 2$. Dashed curve (BCL) is flux for $P_{2}=$ constant (see text).

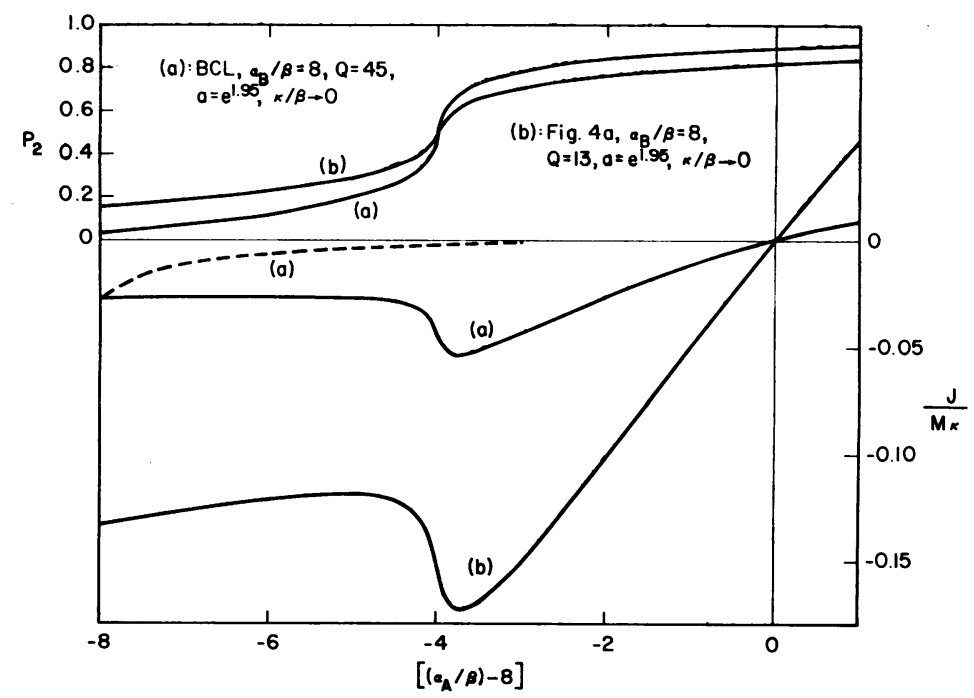

FIG. 3. Cooperative effect on the flux when $\alpha_{B} / \beta=8:(a)$ BlumenthalChangeux-Lefever (BCL) model; $(b)$ model in Fig. 4a. $Q$ chosen (arbitrarily) to give $P_{2}=1 / 2$, when $\alpha_{A}=\alpha_{B} / 2$. Dashed curve (BCL) is flux for $P_{2}=$ constant (see text). 
remained constant at its $\alpha_{A} / \beta=0$ value. It is thus clear that there are two effects working in opposite directions, as $\alpha_{\mathrm{A}} / \beta$ increases: (1) the flux per unit of type II decreases in absolute value (dashed line); but (2) the number of units of type II increases even faster (in these examples).

The equilibrium equation (10) shows very clearly the influence of binding 0 molecules on I-II relative stability. If $Q>1$, type I is favored $\left(P_{2}<1 / 2\right)$, when $\lambda=0(y=1 / Q<1)$. But, on increasing $\lambda, P_{2}$ increases: $P_{2}=1 / 2$ when $y=1 ;$ and $P_{2}>1 / 2$ for $y>1$. Thus, type II will always predominate, for any $Q$, if $\lambda$ is sufficiently large.

We do not show any "phase transition" curves $\left(a>e^{2}\right)$ in Figures 2 and 3 because: (1) the experimental potassium flux curves are "subcritical" (i.e., they suggest cooperativity, but not to the extent of a sharp transition); and (2) the Bragg-Williams approximation (in the above form) is inadequate, even qualitatively, if there is an actual transition (see below).

Simplifications of the Blumenthal-Changeux-Lefever Model. The model can be simplified (see Figs. $4 a$ and $4 b$ ) without alteration of the qualitative be-

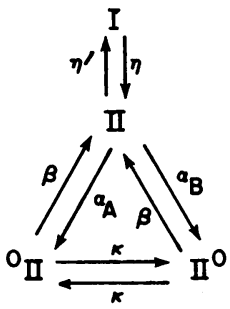

a

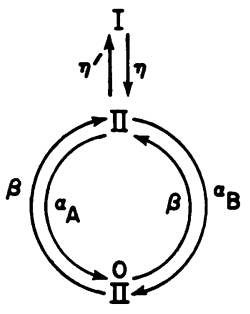

b

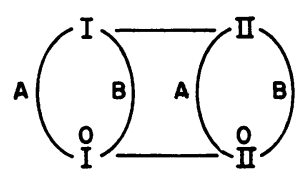

FIG. 4.- (a) (b) Simplifications of the model in Fig. $1 b$ which have the same qualitative properties. (c) Generalization of $(b)$ which is used in part III. Line = pair of arrows.

havior discussed above. In Figure $4 a$, only one molecule of 0 can be bound on a type II unit, although there are still two sites; ${ }^{14}$ in Figure $4 b$, there is only one (symmetrically placed) site.

In the former case (Fig. $4 a$ ), application of the diagram method ${ }^{9}$ leads to the following steady-state properties:

$$
\begin{gathered}
p_{1}=\eta^{\prime} \beta(2 \kappa+\beta) / \Sigma, \\
p_{2}=\eta \beta(2 \kappa+\beta) / \Sigma, \\
p_{02}=\eta\left[\kappa\left(\alpha_{\mathrm{A}}+\alpha_{\mathrm{B}}\right)+\alpha_{\mathrm{A}} \beta\right] / \Sigma, \\
p_{20}=\eta\left[\kappa\left(\alpha_{\mathrm{A}}+\alpha_{\mathrm{B}}\right)+\alpha_{\mathrm{B}} \beta\right] / \Sigma, \\
\Sigma=(2 \kappa+\beta)\left[\eta\left(\alpha_{\mathrm{A}}+\alpha_{\mathrm{B}}+\beta\right)+\eta^{\prime} \beta\right], \\
P_{2} a^{1-2 P_{2}} /\left(1-P_{2}\right)=\left[\left(\alpha_{\mathrm{A}} / \beta\right)+\left(\alpha_{\mathrm{B}} / \beta\right)+1\right] / Q,
\end{gathered}
$$

and

$$
\frac{J}{M \kappa}=\frac{P_{2}\left[\left(\alpha_{\mathrm{A}} / \beta\right)-\left(\alpha_{\mathrm{B}} / \beta\right)\right]}{[1+2(\kappa / \beta)]\left[\left(\alpha_{\mathrm{A}} / \beta\right)+\left(\alpha_{\mathrm{B}} / \beta\right)+1\right]} .
$$

Note that $P_{2}$ does not depend on $\kappa$. 
In the latter case (Fig. $4 b$ ),

$$
\begin{gathered}
p_{1}=2 \eta^{\prime} \beta / \Sigma, p_{2}=2 \eta \beta / \Sigma, p_{02}=\eta\left(\alpha_{\mathrm{A}}+\alpha_{\mathrm{B}}\right) / \Sigma, \\
\Sigma=\eta\left(\alpha_{\mathrm{A}}+\alpha_{\mathrm{B}}+2 \beta\right)+2 \eta^{\prime} \beta, \\
P_{2} a^{1-2 P_{2}} /\left(1-P_{2}\right)=\left[\left(\alpha_{\mathrm{A}} / \beta\right)+\left(\alpha_{\mathrm{B}} / \beta\right)+2\right] / 2 Q, \\
\text { and } J / M \beta=P_{2}\left[\left(\alpha_{\mathrm{A}} / \beta\right)-\left(\alpha_{\mathrm{B}} / \beta\right)\right] /\left[\left(\alpha_{\mathrm{A}} / \beta\right)+\left(\alpha_{\mathrm{B}} / \beta\right)+2\right] .
\end{gathered}
$$

It is apparent that the $P_{2}$ and $J$ curves will have the same appearance in these two cases, except for scale factors in $\alpha / \beta$ and $J$. Equations (15) and (16) are illustrated in Figures 2 and 3.

Because of the simplicity of equations (17) to (20), it is easy to treat the generalization of Figure $4 b-4 c$. This is done in part III, including membrane potential effects. In fact, this generalization proves to be essential in order to duplicate the appearance of experimental curves (flux vs. membrane potential).

Comments on the Bragg-Williams Approximation. There is a logical inconsistency in the way we introduced the Bragg-Williams approximation into the grand partition function of a unit in equation (7) and into $\eta^{\prime} / \eta$, and hence the kinetics, in equation (8). That is, the Bragg-Williams factors are appropriate for a closed system, but the present system is an open one (not only with respect to 0 , but more especially in that $N \equiv P_{2} M$, the number of type II units, can fluctuate). This distinction is inconsequential, however, except in the neighborhood of a phase transition.

The Bragg-Williams approximation in an open equilibrium system has been discussed in detail elsewhere. ${ }^{15,}{ }^{16}$ Also pertinent is the stochastic treatment of a simple steady-state system, ${ }^{17}$ since the kinetics of the complete probability distribution in $N, P(N)$, must be considered at a phase transition, not simply the mean value $\bar{N}$.

Because the steady-state equations (15) and (19) are also appropriate for a system in equilibrium with an average bath, we may surmise with some confidence that the steady-state distribution $P(N)$, in these two cases, has the equilibrium form. ${ }^{17}$ We can make the same assumption with respect to equation (11), but with somewhat less assurance. ${ }^{18}$ Then if we denote the right-hand side of any of these three equations by $y$, we have $\mathrm{e}^{15,16}$

$$
\begin{gathered}
P(N)=\text { const } \times M ! y^{N} a^{[N-(M / 2)]^{2 / M}} / N !(M-N) ! \\
\bar{N}(y)=\sum_{N=0}^{M} N P(N), \bar{P}_{2}(y)=\bar{N}(y) / M .
\end{gathered}
$$

Unlike $P_{2}(y)$ in equations (11), (15), and (19), $\bar{P}_{2}(y)$ does not exhibit ${ }^{15}, 16$ a "loop" when $a>e^{2}$ (phase transition). If $M$ is very large, $P_{2}$ shows an abrupt jump at $y=1$; if $M$ is of the order of several hundred or less, there is appreciable rounding off of the discontinuity.

The above paragraph describes the stable steady-state behavior of an open system (averaging over a very long period of time). In a real experiment, however, one is likely to encounter metastability [the system is confined to one of the two peaks in $P(N)$ ] which persists until nucleation of the stable phase occurs. 
In this case, the metastable parts of the Bragg-Williams loop are to be used. However, the unstable part of the Bragg-Williams loop has no similar validityit is merely the locus of the minimum in $P(N)$ between two peaks. ${ }^{15}, 16$

Incidentally, nucleation ${ }^{6}$ cannot be investigated within the framework of the Bragg-Williams approximation alone because it is implicit in this approximation that, for any $N$, there is a uniform density, $N / M$, throughout the entire system.

All of the flux equations, above, have the form $J=$ flux per type II unit $\times$ $P_{2} M$. In the open system modification just discussed, we merely replace $P_{2}$ by $\bar{P}_{2}$.

A practical exact approach to the cooperative problems in this series, including metastability and nucleation, is by computer simulation ${ }^{4}$ on a small system with periodic boundary conditions. We have initiated work of this kind, especially on the oscillatory phase transition problem discussed in part II.

We are very much indebted to Professor Max Delbrück for bringing the Blumenthal, Changeux, and Lefever paper to our attention, and for a valuable discussion.

* Supported in part by research grants from the General Medical Sciences Institute of the U.S. Public Health Service and the National Science Foundation.

${ }^{1}$ Hill, T. L., and I. W. Plesner, J. Chem. Phys., 43, 267 (1965).

${ }^{2}$ Hill, T. L., and O. Kedem, J. Theoret. Biol., 10, 399 (1966).

3 Hill, T. L., these Proceedings, 58, 111 (1967). In equation (15) of this paper, the two left-hand exponents should read $-c w_{A A} / 2 k T$ and $-c w_{B B} / 2 k T$.

4 Gordon, R., J. Chem. Phys., 49, 570 (1968).

- Blumenthal, R., J.-P. Changeux, and R. Lefever, Comptes Rendu, 270D, 389 (1970); J. Membrane Biol., to be published.

6 Adam, G., Z. Naturf., 23, 181 (1968); Ber. d. Bunsengesellschaft, 71, 829 (1967); Zeitschr. f. Naturforsch., 23b, 181 (1968); Proceedings of the Conference on Physical Principles of Biological Membranes, Coral Gables, December 18-20, 1968, ed. A. Perlmutter (Gordon and Breach Publishing Co., in press); Zeitschr. Physik. Chemie N.F., in press.

7 Ehrenstein, G., and D. L. Gilbert, Biophysical J., 6, 553 (1966), Figures 1 and 7.

${ }^{8}$ Cole, K. S., Membranes, Ions and Impulses (Berkeley: University of California Press, 1968), p. 458.

${ }^{9}$ Hill, T. L., Thermodynamics for Chemists and Biologists (Reading, Mass.: AddisonWesley Publishing Co., 1968), section 7.5.

${ }_{10}$ Hill, T. L., these Proceedings, 55, 1379 (1966); see also reference 2, Model 2.

11 Hill, T. L., Introduction to Statistical Thermodynamics (Reading, Mass.: AddisonWesley Publishing Co., 1960), chap. 14.

${ }^{12}$ See equation (14-56) of reference 11.

${ }_{13}$ See Figure 1, reference 3, or Figure 14-5, reference 11.

14 See reference 2, Model 3.

15 Hill, T. L., J. Phys. Chem., 57, 324 (1953).

${ }^{16}$ Hill, T. L., Statistical Mechanics (New York: McGraw-Hill Book Co., 1956), appendix 9.

${ }_{17}$ Reference 9, Section 7.3.

18 This is an important question, and one which we are investigating. 